\title{
An Indigenous Removal Method of a Broken Interlocking Nail
}

\author{
${ }^{1}$ Aniket Kedawat, ${ }^{2}$ AK Mathur, ${ }^{3}$ Mohit Kumar, ${ }^{4}$ Anshu Sharma, ${ }^{5}$ Anand Gupta
}

\begin{abstract}
Breakage of locking nail is an important cause of interlocking nail failure in femoral fractures. Usually, it takes place at one of the ends of the nail. Here we report an unusual extraction method of intramedullary femoral nail failure with breakage of nail proximal to distal locking holes. Such a scenario usually complicates further management. This case was managed with exchange nailing and bone grafting after removal of implant using a novel technique and using commonly available orthopedic instruments in the operation theater. Here, we briefly review the literature regarding such an unusual presentation and discuss in detail the possible etiology of such a presentation and the management options when facing such a complex situation.
\end{abstract}

Keywords: Broken nail, Exchange nail, Implant removal.

How to cite this article: Kedawat A, Mathur AK, Kumar M, Sharma A, Gupta A. An Indigenous Removal Method of a Broken Interlocking Nail. J Mahatma Gandhi Univ Med Sci Tech 2017;2(1):38-40.

\section{Source of support: Nil}

\section{Conflict of interest: None}

\section{INTRODUCTION}

Intramedullary nails used for femur fixation will eventually break if the fracture does not unite within the stipulated duration for fracture union. An exchange nailing as a definitive method can be performed when a fracture has not united but healing process is ongoing as seen on radiographs. This is best conducted without interfering with the callus and overlying soft tissues. Here we report a case in which the medullary nail broke near the distal locking screws.We used a Steinmann pin and a T-handle to remove the broken nail and then we did exchange nail and bone grafting.

\section{CASE REPORT}

A male patient, 34 years old, weighing $106 \mathrm{~kg}$ had closed and comminuted fracture of femur at proximal and middle

\footnotetext{
${ }^{1,5}$ Resident (2nd Year), ${ }^{2}$ Professor and Unit Head, ${ }^{3}$ Associate Professor, ${ }^{4}$ Resident (3rd Year)

${ }^{1-5}$ Department of Orthopaedics, Mahatama Gandhi National Institute of Medical Sciences, Jaipur, Rajasthan, India

Corresponding Author: Aniket Kedawat, Resident (2nd Year) Department of Orthopaedics Mahatama Gandhi National Institute of Medical Sciences, Jaipur, Rajasthan, India, Phone: +919636196015, e-mail: aniketkedawat@gmail.com
}

one-third junction on the left side resulting from motorcycle accident. There were no other injuries. We used a Cyrus nail of length $400 \mathrm{~mm}$ (11 mm) after closed reaming up to $12 \mathrm{~mm}$, locked proximally with three screws and distally with two screws and open circumference wiring and cancellous bone grafting. There were no postoperative complications. The patient was allowed mobilization using crutches with toe touch partial weight bearing. He did not come for a regular follow-up, and after 3 months he presented in emergency room with sudden severe onset of pain at fracture site and was not able to bear weight on the affected side. Patient had started full weight bearing against our advice. Plain radiographs revealed break of nail just proximal to distal locking screws (Fig. 1).

Patient was admitted for the revision surgery (exchange nailing with encirclage and bone grafting). The proximal nail fragment was removed using a standard technique through the original entry point. We used a lateral incision over left distal thigh for the distal fragment. The distal locking screws were not removed at this point. The distal fracture fragment was exposed, curetted, and using a T-handle and $3.5 \mathrm{~mm}$ Steinmann pin, we inserted this into the distal nail fragment and using cork screw maneuver we jammed it into the distal fragment. Once jammed we pulled out the assembly and extricated the nail fragment. Following removal of the broken nail and all screws, an exchange nailing procedure was done supplemented with circumferential wiring and bone grafting (Fig. 2).

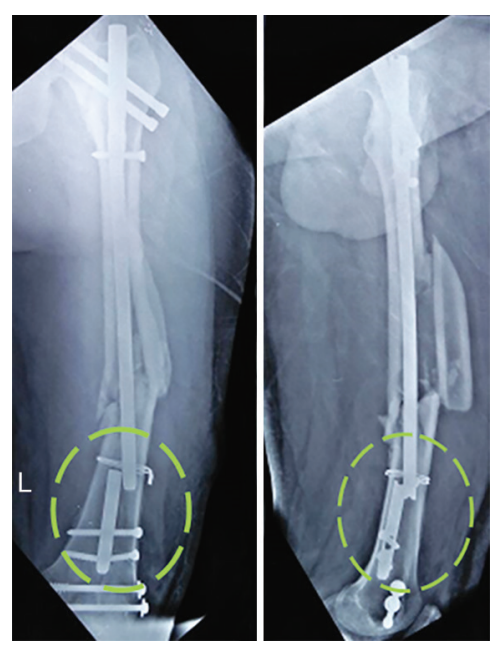

Fig. 1: Broken implant 

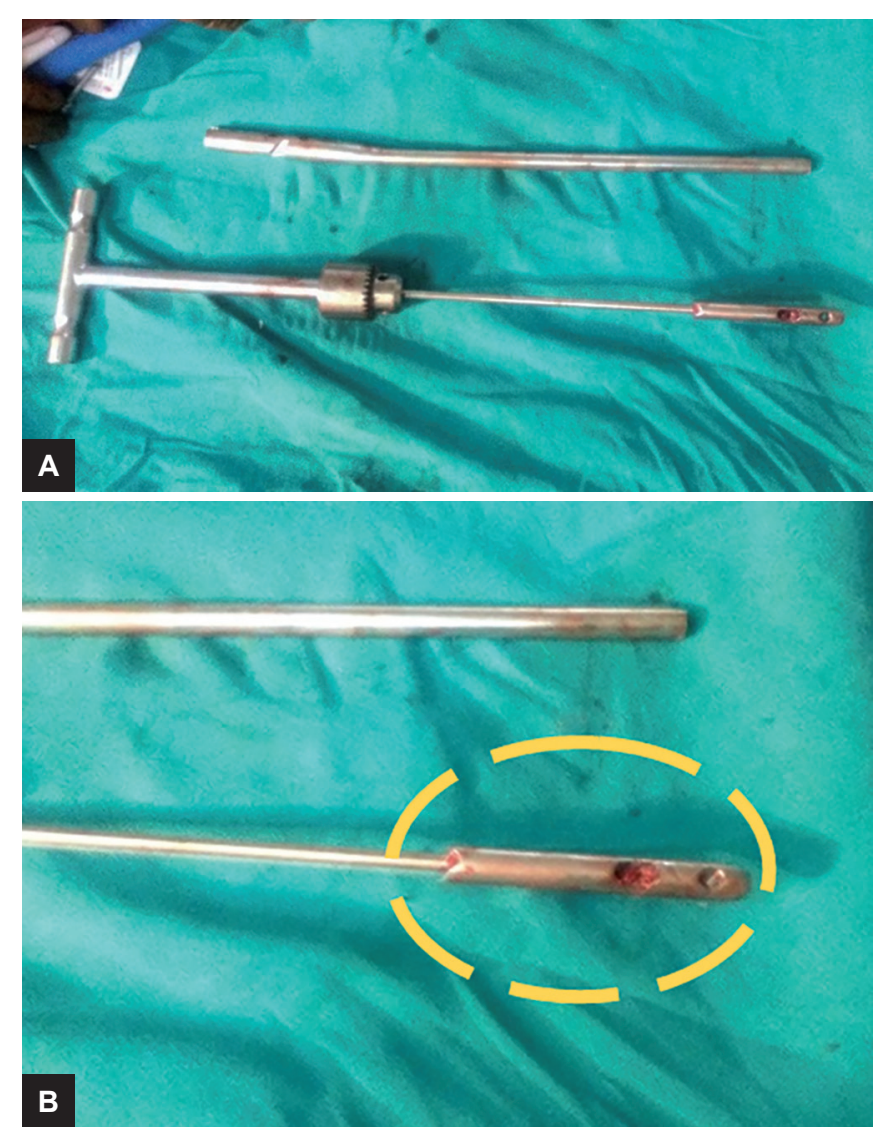

Figs 2A and B: Intraoperative broken implant attached with (A) T-handle; and (B) Steinmann pin

The patient was mobilized with partial weight bearing with the help of crutches. Gradually, full weight bearing was allowed 3 months onward. Quadriceps strengthening and knee mobilization exercise were started as per standard rehabilitation protocol (Figs 3 and 4).

\section{DISCUSSION}

Femur is the largest bone of the body and one of the main load-bearing bones of the lower limb. Shaft of femur fractures are common and often associated with highenergy injuries. Various treatment methods are available. Intramedullary nail without interlocking screws is not routinely practiced because it has inadequate immobilization of fracture due to rotational instability. Using interlocking screw bolts at proximal and distal ends of the nail greatly improves rotational stability, but it produces high stress concentrations at the ends of the nail. The reported rate of nail breakage varies from 0.5 to $3.3 \%$ in different studies. Fatigue failure of intramedullary nails is less frequent nowadays because of advances in nail design and an appreciation for the biomechanics of nails. When the interlocking femoral nail break, it usually indicated fracture not getting united. For all implants stabilizing the fractures, a race goes between healing of the fracture and failure of implant. When a fracture does not heal in

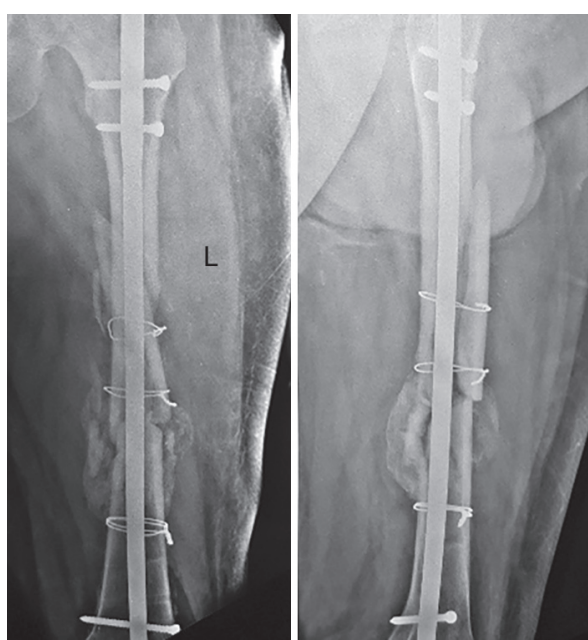

Fig. 3: Three-months postoperative X-ray

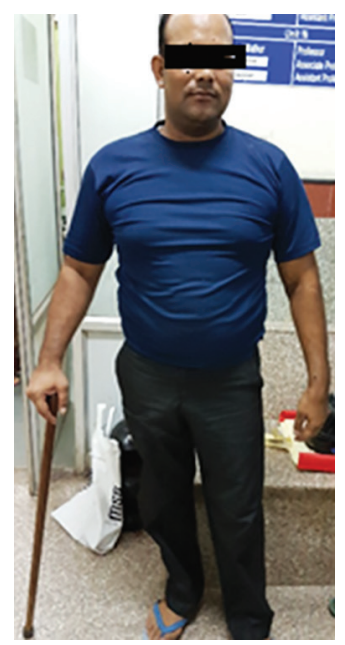

Fig. 4: Three-months postoperative patient

due course of time the implant will get fatigued and will break. The timing of this failure depends on numerous factors, including the size of the nail and its locking bolt, type of metal, fracture pattern, location of the nonunion, patient's weight, timing of starting weight bearing, and patient's activity level. There are several methods described for the extraction of broken intramedullary nails. Franklin et $\mathrm{al}^{1}$ used a technique where custommade hook was used to extract the distal fragment in 56 patients. Hooks of variable sizes were available for the different sized nails. Metikala and Mohammed $^{2}$ described eight patients in whom closed retrograde retrieval of the distal broken segment was done using a ball-tipped guidewire. The technique involved extraction of proximal nail fragment followed by a standard plain guidewire insertion through the cannulated distal broken nail segment. The plain guidewire was pushed distally till knee joint under fluoroscopy imaging. Over this wire, a 5-mm cannulated large drill bit was used to create a track up to the distal broken nail segment. Through the small knee wound using smooth end first, a ball-tipped 
guidewire was passed, till the ball engaged the nail end. The guidewire extraction was done along with the broken nail through the proximal wound. Cabrita et $\mathrm{al}^{3}$ reported 5 patients and used a technique involving the application of an $\mathrm{AO}$ distractor prior to removal of the nail fragments, with subsequent removal of the proximal nail fragment in an anterograde fashion and removal of the distal fragment through a medial para patellar approach. Fracture impaction was then accomplished using a nail that was wider than the remaining fragmented material. These methods have the disadvantage of opening the knee joint unnecessarily. Georgilas et al ${ }^{4}$ used a $10.5 \mathrm{~mm}$ reamer, which was passed over a guidewire into the intramedullary canal and wedged into the distal broken fragment. The nail was removed gently using rotatory movements under fluoroscopic guidance. Blake ${ }^{5}$ in his technique used an olive-tipped guidewire that was passed completely through the nail. Using standard extraction set, proximal part was removed. Then through the distal fragment adjacent to the previously placed wire, another nonolive-tipped wire was passed. The olive-tipped wire was then withdrawn, causing it to impinge against the nontipped wire and the outer tip of the nail. A T-handled chuck was placed at the proximal end of the olive-tipped wire and it was gently tapped back bringing along with it, the distal fragment. But, using a small tapered nail, it may not allow two guidewires to protrude through the distal end simultaneously. In the present case, we made use of tools readily available in an orthopedics operating room. We had no difficulty in removing the distal broken fragment of nail and the procedure was quick to perform. This method adds to the existing methods that a surgeon can use to remove the distal end of a broken nail.

\section{REFERENCES}

1. Franklin JL, Winquist RA, Benirschke SK, Hansen ST Jr. Broken intramedullary nails. J Bone Joint Surg Am 1988 Dec;70(10):1463-1471.

2. Metikala S, Mohammed R. Closed retrograde retrieval of the distal broken segment of femoral cannulated intramedullary nail using a ball-tipped guide wire. Indian J Orthop 2011 Jul;45(4):347-350.

3. de Amorim Cabrita HA, Malavolta EA, Teixeira OV, Montenegro NB, Duarte FA, Mattar R Jr. Anterograde removal of broken femoral nails without opening the nonunion site: a new technique. Clinics (Sao Paulo) 2010 Mar;65(3):279-283.

4. Georgilas I, Mouzopoulos G, Neila C, Morakis E, Tzurbakis M. Removal of broken distal intramedullary nail with a simple method: a case report. Arch Orthop Trauma Surg 2009 Feb; 129(2):203-205.

5. Blake SM. A technique for the removal of the distal part of a broken intramedullary nail. Ann R Coll Surg Engl 2009 Mar;91(2):169-170. 